\title{
Cauda Equina Syndrome
}

National Cancer Institute

\section{Source}

National Cancer Institute. Cauda Equina Syndrome. NCI Thesaurus. Code C35436.

A rare neurologic disorder caused by impingement of the nerve roots of the cauda equina secondary to disc herniation, spinal stenosis, vertebral fracture, neoplasm or infection. Clinical signs may include bladder or bowel dysfunction, paresthesia and weakness of the lower extremities. The clinical course rapidly deteriorates and necessitates emergent surgical decompression to minimize risk of incontinence, sexual dysfunction and permanent paralysis. 